\title{
Indentation size effect and the Hall-Petch 'Law'
}

\author{
L. M. Brown \\ Cavendish Laboratory \\ University of Cambridge \\ J. J. Thomson Ave, Cambridge CB3 OHE \\ <Imb12@cam.ac.uk>
}

Keywords: hardness, plasticity, dislocations, polycrystals

\begin{abstract}
The flow of material out from under regions in compression must occur by the operation of many slip systems, which together produce rotational flow. Such flow requires the accumulation of geometrically necessary dislocations, and leads to the indentation size effect: smaller indents produce higher hardness, a component of the hardness being inversely proportional to the square-root of the indenter size. A pattern of flow in polycrystals which satisfies both continuity of normal stress and continuity of matter at boundaries can be achieved by rotational flow, and it leads to a grain-size effect. Under most circumstances, the flow stress has a component which is inversely proportional to the square-root of the grain size, the Hall-Petch law. The flow is accompanied by the build-up of internal stress which can be relieved by intercrystalline cracking, thereby limiting the cohesive strength of polycrystals. The relationship between these ideas and traditional views is briefly explained, and an analysis is given of recent experimental results.
\end{abstract}

\section{Introduction}

One of the most fruitful developments in modern understanding of plastic flow in terms of dislocations has been Ashby's [1] concept of 'geometrically necessary dislocations'. The idea is that in many circumstances the pattern of plastic flow must of necessity store dislocations which contribute to the stress required to continue flow. The idea has given rise to a well-developed mathematical theory of 'strain-gradient plasticity', widely used to study problems concerning nonuniform plastic flow: see Fleck \& Hutchinson [2]. However, the theory requires at least one microstructural parameter whose identification has caused controversy. Electron microscopists in particular find that the structures predicted by the theory do not really correspond to observation.

Brown [3] suggested using simplified constructions from the theory of plasticity to estimate the storage of dislocations in problems of inhomogeneous plastic strain. These constructions correspond to structures which have been observed for many years by both optical and electron microscopy. A major prediction of this approach is that the hardness of ductile materials increases with decreasing size of indentation, contrary to standard plasticity. For a wedge indenter, the hardness $H$, load applied to the indenter divided by projected area of indentation, should be given by

$$
H_{R}=T\left(\sigma_{0}+\alpha \mu b \sqrt{\rho_{0}+\tan \beta / b d}\right)
$$

Here, $T$ is the 'Tabor factor', the ratio of normal stress to the flow stress in shear under the indenter, a constant with the theoretical value of $2 \pi$, often taken approximately equal to $6 . \sigma_{0}$ is the intrinsic lattice friction stress, usually nearly zero for ductile face-centred metals; $\alpha$ is the forest hardening constant, about equal to $1 / 3 ; \mu$ is the elastic shear modulus; $b$ is the magnitude of the 
Burgers vector, sometimes called the 'Burgers scalar', $\rho_{0}$ is the forest dislocation density at what Tabor called the 'representative strain'; $\tan \beta$ is the effective engineering shear strain (twice the tensor strain) caused by the angle $\beta$ between the indenter surface and the flat surface under it; and $d$ is the width of the indentation. All of these parameters have known or readily measurable values.

Equation (1) is based on a pattern of circular rotational flow. It causes 'pile-up', namely a mound of material in the neighbourhood of the indentation, stretching from the centre out to $3 d / 2$, and it causes physical rotation of the crystal lattice planes, as shown by the lines ABCD in Fig. 1. The maximum angle of rotation is approximately equal to $\beta / 2$. However, rotational flow is not the only response of the material to indentation. Commonly observed, especially for small indentations and indentations in materials with a high friction stress is 'laminar flow'.

Laminar flow, illustrated in Fig. 2, takes place by the punching of dislocation loops in stacks along the available slip directions. Where the loops meet the surface, etch pits can be seen, resulting in the famous 'rosette' patterns studied for many years by optical methods. The loop stacks extend very far from the indentation, much further than the plastic zone of rotational flow.

A review by Chaudhri [4] presents a satisfactory analysis of the extent of loop stacks in many materials. The major implicit assumption of the analysis is that the hardness is independent of the indentation size. This type of flow can result in 'sink-in', that is to say, the depression of material outside the indenter, driven by the load. It is likely that the load required producing this

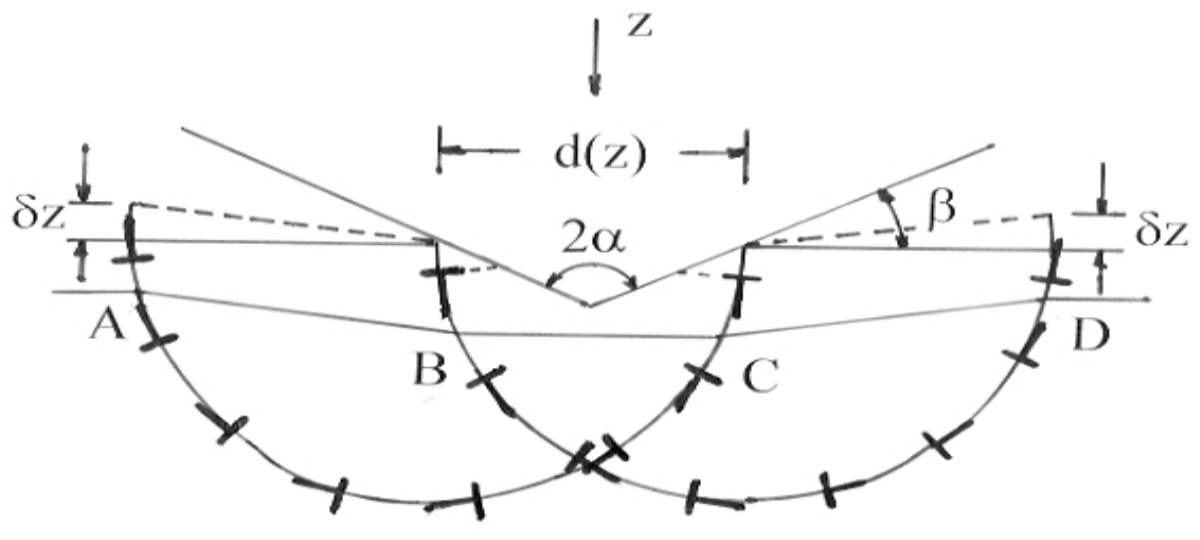

Fig. 1. A wedge indenter penetrates a flat surface in the $z$ direction, causing rotational flow, pushing material out from under it. The flow is postulated to occur around a circle, the 'slip circle', and as a result geometrically necessary dislocations are stored as shown. They form a small-angle boundary and are residual dislocations resulting from shear on the several different intersecting slip planes under the indenter. Each infinitesimal increase in depth of indentation, $\delta z$, causes an infinitesimal increment in the rotation $\delta z / d(z)$ of the lattice planes and thus an increase in the number of dislocations whose spacing on the circle is inversely proportional to the diameter of the circle, and hence to the size of the indentation. Thus there is an indentation size effect. 


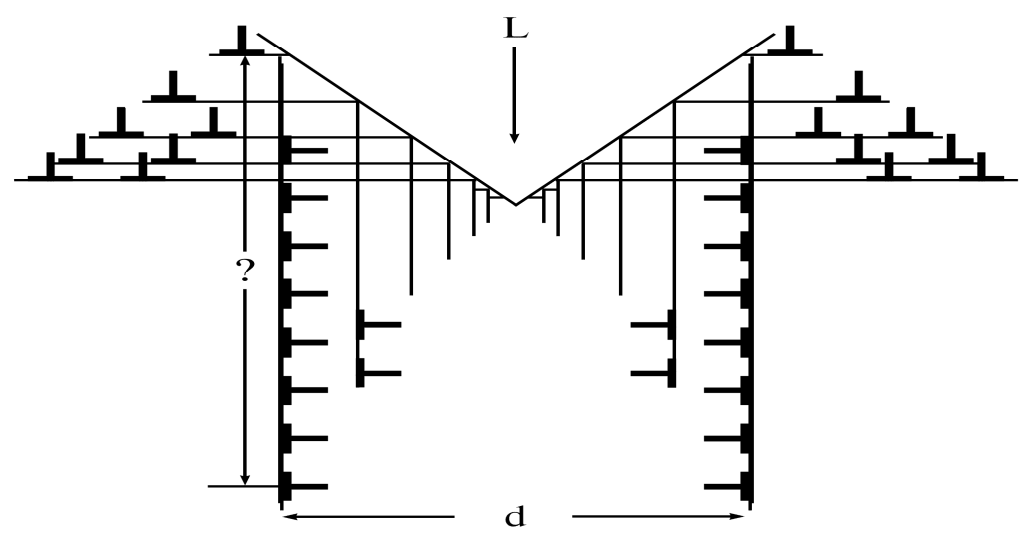

Fig. 2 shows schematically the production of loop stacks under a wedge indenter. They do not cause overall lattice rotation, nor any pile-up of material at the surface, but they do produce elastic strain from the storage of excess matter under and around the indentation. The length of the loop stacks is very variable. It may extend to a hundred times the indent size. Optical microscopy of the etched structure reveals a pattern of loops called a 'rosette' pattern.

flow has a complex relationship with the depth of the indentation, fluctuating sharply with the initiation of each dislocation loop or loop stack, so-called pop-in phenomena, but a qualitative analysis [5] suggests that on average, for laminar flow,

$$
H_{L}=C \sigma_{F}
$$

The value of $C$, the 'Chaudhri constant', is empirically found [3] to be about 36 , six times the Tabor constant.

The pattern of laminar flow is nearly orthogonal to that for rotational flow. One can imagine that the two patterns act as independent channels of material response, so that laminar and rotational hardness can be combined in parallel to produce the total hardness:

$$
\frac{1}{H}=\frac{1}{H_{L}}+\frac{1}{H_{R}}
$$

The theory has been developed for a range of circumstances. Czerski and Brown [5] find for the hardness due to rotational flow under a spherical indenter of radius $r$, producing a circular indentation of radius $a$,

$$
H_{R}=T\left(\sigma_{0}+\alpha \mu b \sqrt{\rho_{0}(\varepsilon)+\frac{1-\left(a^{2} / 4 r^{2}\right)}{4 b r}}\right)
$$

As in eq. (1), the dislocation density $\rho_{0}(\varepsilon)$ denotes the forest density controlling the tensile flow stress $\sigma$ at the representative tensile strain $\varepsilon$, given according to Tabor [6] by $\varepsilon \approx 0.18 \mathrm{a} / \mathrm{r}$. The remarkable prediction of this equation is that it predicts an indenter size effect, but not an indentation size effect for small representative strains. 
Equation (1) accounts quantitatively for early observations using a Vickers indenter in tungsten [7], equations (1), (2), and (3) account for similar work extending to the nanometre size range using a Berkovich indenter in iridium [3,8], and equations (2), (3), and (4) account quantitatively [5] for the observations of Lim and Chaudhri [9] on work-hardened oxygen-free copper. The value of the Chaudhri constant was taken to be 36 throughout these analyses. There are no fitting parameters, apart from the Chaudhri constant and the hardness at large indentation size (the background hardness) independently determined.

In a further development, Brown [10] has re-analysed the hardening due to rotational flow induced by the uniaxial straining of polycrystals with grain size $L$ to find

$$
\sigma=\sigma_{0}+M \alpha \mu b \sqrt{\rho(\varepsilon)+\frac{A \varepsilon}{L}}
$$

where $M$ is the Taylor factor, about equal to 3, which converts the flow stress in shear to the tensile flow stress of a polycrystal at plastic uniaxial strain $\varepsilon$. The constant $A$ results from averaging the Schmidt factors $S$ of the polycrystalline array and for planar (non-wavy) glide in f.c.c. and b.c.c. ductile crystal structures is given by

$$
A=\frac{\pi}{b} \sqrt{\frac{\left\langle S^{4}\right\rangle-\left\langle S^{2}\right\rangle^{2}}{\left\langle S^{2}\right\rangle^{2}}} \cong \frac{0.53}{b}
$$

The picture underlying this analysis is of the grains interacting one with another in such a way that grains in a soft orientation, extended by plastic tensile strain more than the average, transfer material to grains of a hard orientation, less extended. Detailed comparison of equation (6) with the excess dislocation density caused by rotational flow suggests that at room temperature in nickel it is underestimated by a factor of about six. Narutani and Takamura [11] accurately measured dislocation density using electrical resistance. They show that the 'geometrically necessary' dislocation density in fact contains a mildly temperature-dependent component, decreasing with increasing temperature, indicative of processes such as cross-slip and local climb which can eliminate surplus statistically stored dislocations at higher temperatures. Only the density of residual geometrically necessary dislocations should be independent of temperature.

It is easiest to understand the behaviour of indenters which display self-similarity as the depth of the indentation increases. Fig. 3 shows how the hardness is expected to vary with indentation size under a wedge indenter. Rotational flow produces an increment which is largely geometrically determined. It is independent of the state of work-hardening and the friction stress due to solution hardening or to intrinsic lattice effects. It depends upon the material only through the magnitude of the Burgers vector and the forest hardening, neither of which vary much from one material to another. For very small indents, laminar flow predominates, and for large indents the geometrically induced dislocations are swamped by the forest density arising from work-hardening either at the representative strain due to the indentation or from prior straining of the bulk material. One can see simply from the diagram how laminar flow predominates for virgin materials and small indentations, whereas rotational flow predominates for heavily work-hardened materials and larger indentations. 'Sink-in', characterised by indents showing concave sides, is easily understood as a consequence of laminar flow, whereas 'pile-up' which produces convex sides, results from rotational flow. There are many observations of this, most notably perhaps the contrast between work-hardened and annealed copper [9]. 


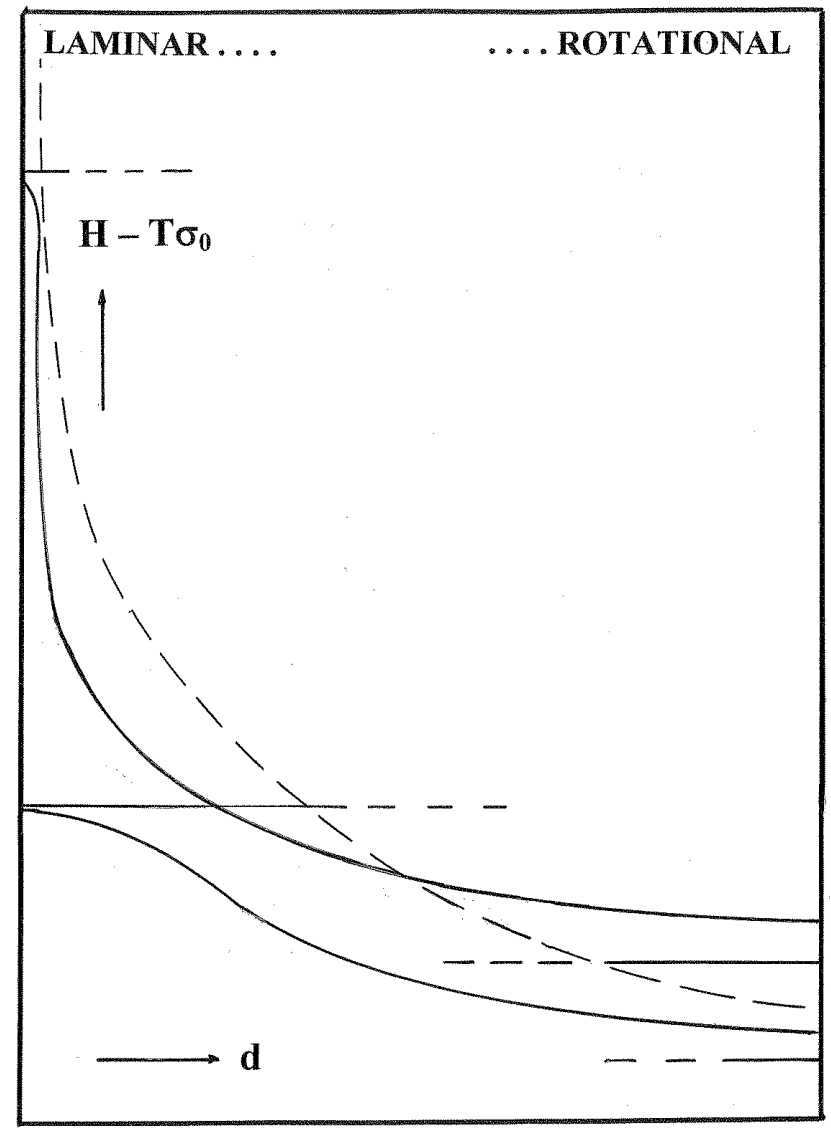

Fig. 3 shows schematically the hardness attributable to dislocations as a function of indent size under a wedge indenter. The flow changes from predominantly laminar to predominantly rotational as the indent size increases. For a work-hardened sample, upper solid curve, the region of laminar flow is restricted to small indents, whereas for a virgin sample, lower solid curve, laminar flow extends to much larger ones. The size-dependent term, which does not depend markedly upon the material, is shown as a broken line. Theory shows that the combined hardness crosses the sizedependent hardness curve at a value 1.28 times the background hardness, so the proportion of rotational flow progressively increases as the background hardness increases. For purposes of illustration in the diagram, the work-hardened sample is assumed to have background hardness three times that of the virgin one; in practice the ratio may be much larger, making the contrast between the two curves more prominent. In the laminar regime, rosettes are found in etched samples, as well as 'sink-in'. Because 'sink-in' lowers the surface outside the edges of the indenter, the indentation develops concave sides. In the rotational regime, a dense cluster of etch pits near the indent is seen, as well as 'pile-up' which produces convex indentation sides.

The theory so far presented is two-dimensional. It is easily extended to three dimensions, although some extra assumptions are needed to do this, as follows:

(i) Each edge of the indentation acts as an axis for rotational flow on slip around it. Residual geometrically necessary dislocations are produced by the resulting misorientation. For example, screws are produced at twist boundaries at the ends of a rotating cylinder, with edge dislocations connected to them running parallel to the axis of the cylinder along its length. Each edge of the indenter thus produces a pile-up zone which mirrors the indent in that axis. In the case of circular indentations, each infinitesimal element of arc acts as an axis. This assumption relies upon the fact that in fully ductile materials rotational flow can occur around any axis [10]. The patterns of pile-up 
produced by these flows are sketched in Fig. 4. Experimental observation shows that such patterns approximate to what is found, although crystallographic effects skew the patterns considerably [7].

(ii) The dislocation density is calculated as line-length per unit volume, rather than as number per unit area. This enables the geometrically necessary density of screw, edge and mixed dislocations to contribute to the estimated overall density.

(iii) The zone directly beneath the indenter where the dislocation distributions overlap can be treated in two ways: either it contributes to the overall average density, or it acts as a non-deforming 'dead zone' and only the non-overlapping regions contribute. Because of the large density there, it seems best to treat it as a dead zone. This amounts to ignoring the overlap region and taking the density within the cylinder of each rotation axis to be the relevant geometrically necessary density.

The three-dimensional formulae are listed in Table 1.

\section{Further comparison with recent experimental data}

(1) The work of Yang, Peng, and Okazaki [12]: These authors used spherical diamond indenters of radii ranging from $0.01 \mathrm{~mm}$ to $0.5 \mathrm{~mm}$ to indent annealed aluminium polycrystals with a grain size larger than the largest indentation. They showed that although the stress-strain curves derived from indentation display similar strain-hardening behaviour regardless of the size of the indenter, nevertheless the position of the curves shows a strong indenter size effect. Their analysis of the results shows that a component of the hardness increases inversely with the square-root of the indenter size. Here we briefly re-analyse their results using equations (2), (3), and (4). Fig. 5 shows the square of the hardness as a function of the inverse indenter size, following equation (4), with $\sigma_{0}=0$, as expected for Al. If the pattern of flow were only rotational, this plot should produce straight lines crossing the ordinate at points corresponding to the work-hardened flow stress at different representative strains. By comparison with the slope predicted from equation (4), it is clear that although the hardness produced by smaller indenters has a component following the expected rotational behaviour, deviations can be seen for the larger indenters. To try to identify the various contributions to the hardness, we look at one indenter size, $0.2 \mathrm{~mm}$, at a range of indentation strains. Firstly, we identify the hardness produced at each strain by the largest indenter as the base hardness. The base hardness values depend upon strain in a manner consistent with macroscopic tensile testing. Secondly, we plot the predicted rotational hardness, equation (4). Then, using the flow stress deduced from the base hardness, we calculate the hardness produced by pure laminar flow. The reciprocal of the sum of the reciprocal rotational and laminar hardnesses gives us an estimate of the total hardness caused by the geometrically necessary dislocations. Finally, we add the components to predict the total hardness, and compare that with what is observed: good agreement is found. The method of analysis employs no parameters which have not been used successfully in previous analyses. If the analysis is sound, we see that of the total observed hardness, about onethird comes from geometrically necessary dislocations. The pattern of flow is mostly rotational, with only a very small component of laminar flow, if any.

The point of this analysis is to answer an important question: under what conditions can the tensile stress-strain curve be easily determined using spherical indenters? For this to be the case, the corrections due to geometrically necessary dislocations should be small. A rule of thumb is that the flow stress due to them should be less than the nominal flow stress in uniform tension or compression, leading to equation (7): 

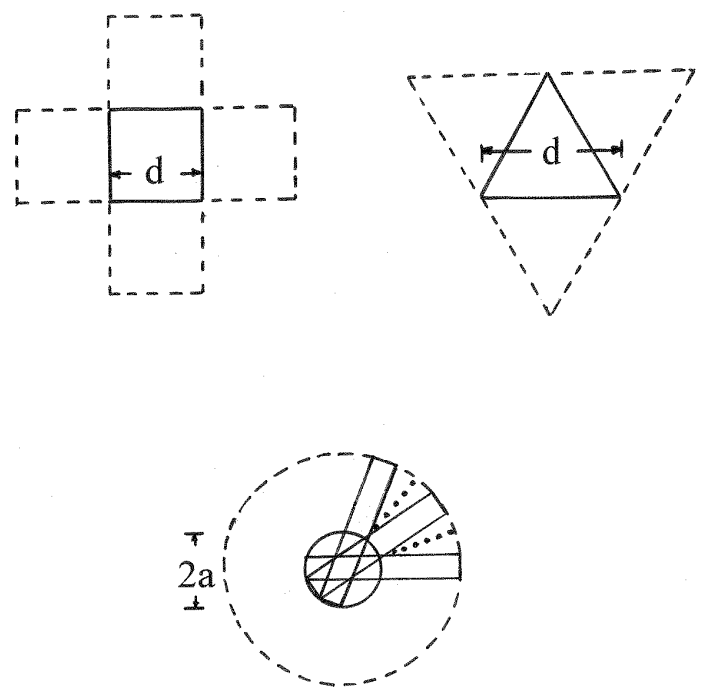

Fig. 4 shows the outline of the indent (solid lines) and the outline of the pile-up (dashed lines) for Vickers, Berkovich, and spherical or conical indenters. Each solid line acts as the axis of a cylinder of rotation. In the case of the circular indent, each infinitesimal element acts as the axis of a cylinder which rotates about it. The outline of three such cylinders is shown. Neighbouring cylinders are imagined to connect along the dotted lines outside the indent, where the residual dislocation line length resulting from the relative rotation of the cylinders is calculated. Within the indent the rotating cylinders overlap.

Table 1 below shows formulae for the 3-D cases, which we shall now use to analyse recent data.

\begin{tabular}{|c|c|c|c|}
\hline Indenter type & 2-D density & 3-D density & $(\alpha, C)$ and remarks \\
\hline $\begin{array}{c}\text { Vickers } \\
\left(\beta=22^{0}\right) \\
\text { square side } d\end{array}$ & $\frac{\tan \beta}{b d}$,eq. (1) & $\frac{\tan \beta}{b d}$ & $\begin{array}{l}\text { ( } 0.26,-) \text { Tungsten single crystal } \\
(111) \text { surface normal }[7] \\
(0.44,-) \text { Tungsten single crystal } \\
(100) \text { surface normal }[7] \\
(0.26,-) \text { annealed gold }[16,17] \\
(0.28,-) \text { annealed nickel }[16,18]\end{array}$ \\
\hline $\begin{array}{c}\text { Berkovich } \\
\left(\beta=25^{0}\right) \\
\text { triangle side } d\end{array}$ & - & $\frac{4 \tan \beta}{\sqrt{3} b d}$ & $\begin{array}{l}(0.22,36) \text { annealed iridium }[8] \\
(0.22,-) \text { Tungsten single crystal } \\
(100) \text { surface normal [19] }\end{array}$ \\
\hline $\begin{array}{l}\text { Square grain, side } \\
L, \\
\text { in polycrystal, } \\
\text { tensile strain } \varepsilon\end{array}$ & $\frac{A \varepsilon}{L}$, eq. (5) & $\frac{3 A \varepsilon}{L}$ & $\begin{array}{l}\text { 3-D formula appears to } \\
\text { underestimate density by about } \\
2 \text { at room temperature [10]. } \\
(0.2,-) \text { NC nickel }[20] .\end{array}$ \\
\hline $\begin{array}{l}\text { Conical, indent } \\
\text { diameter } d\end{array}$ & - & $\frac{3 \tan \beta}{b d}$ & 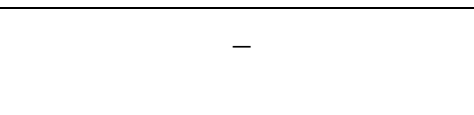 \\
\hline $\begin{array}{l}\text { Spherical indenter, } \\
\text { radius } r \text {, indent } \\
\text { radius } a<<r .\end{array}$ & $\frac{1-\left(a^{2} / 4 r^{2}\right)}{4 b r}$ & $\frac{1-\left(a^{2} / 4 r^{2}\right)}{b r}$ & $\begin{array}{l}(0.25,-) \text { annealed } \mathrm{Al}[12] \\
(0.21,36) \text { work-hardened } \mathrm{Cu} \\
{[5,9]} \\
(0.16,-) \text { annealed } \mathrm{Cu}[13,14]\end{array}$ \\
\hline
\end{tabular}




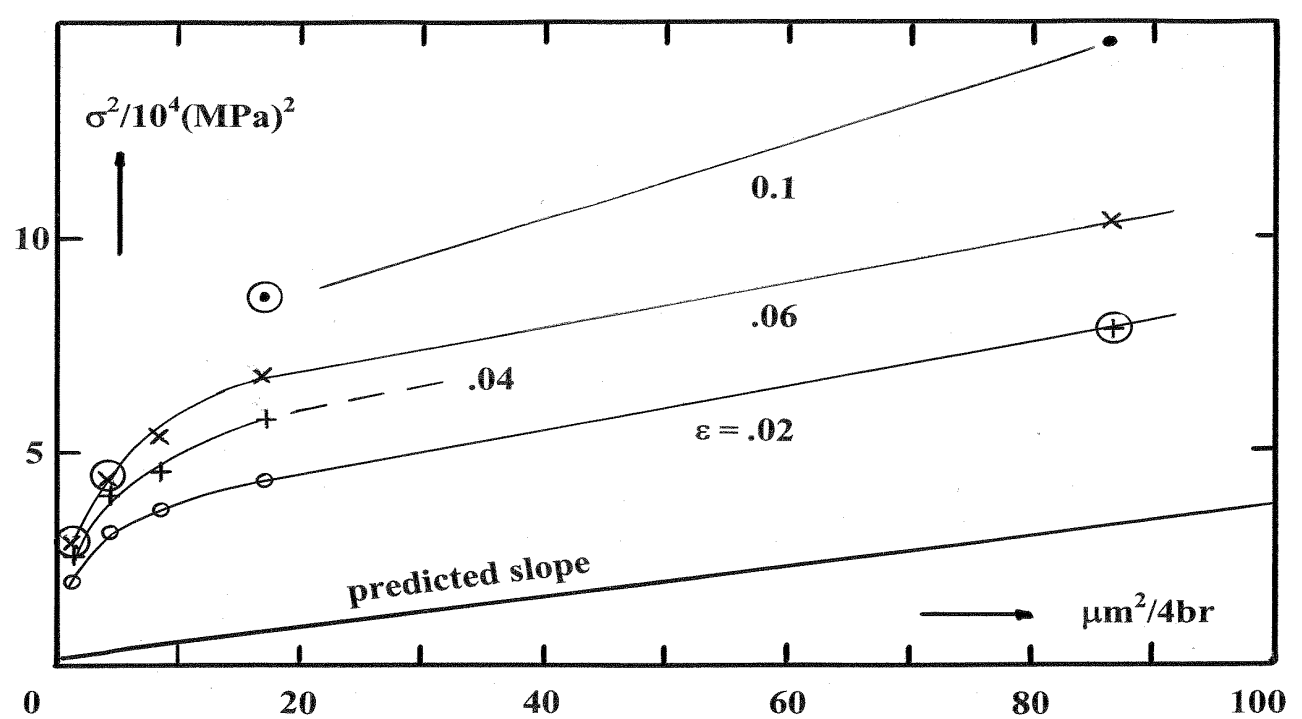

Fig. 5 shows the results of Yang et. al. [12] replotted in accord with equation (4). The values of the strain refer to different indentation radii according to $\varepsilon=.2 a / r$, where $r$ is the indenter radius. Circled points are derived by slight extrapolation from the published data.

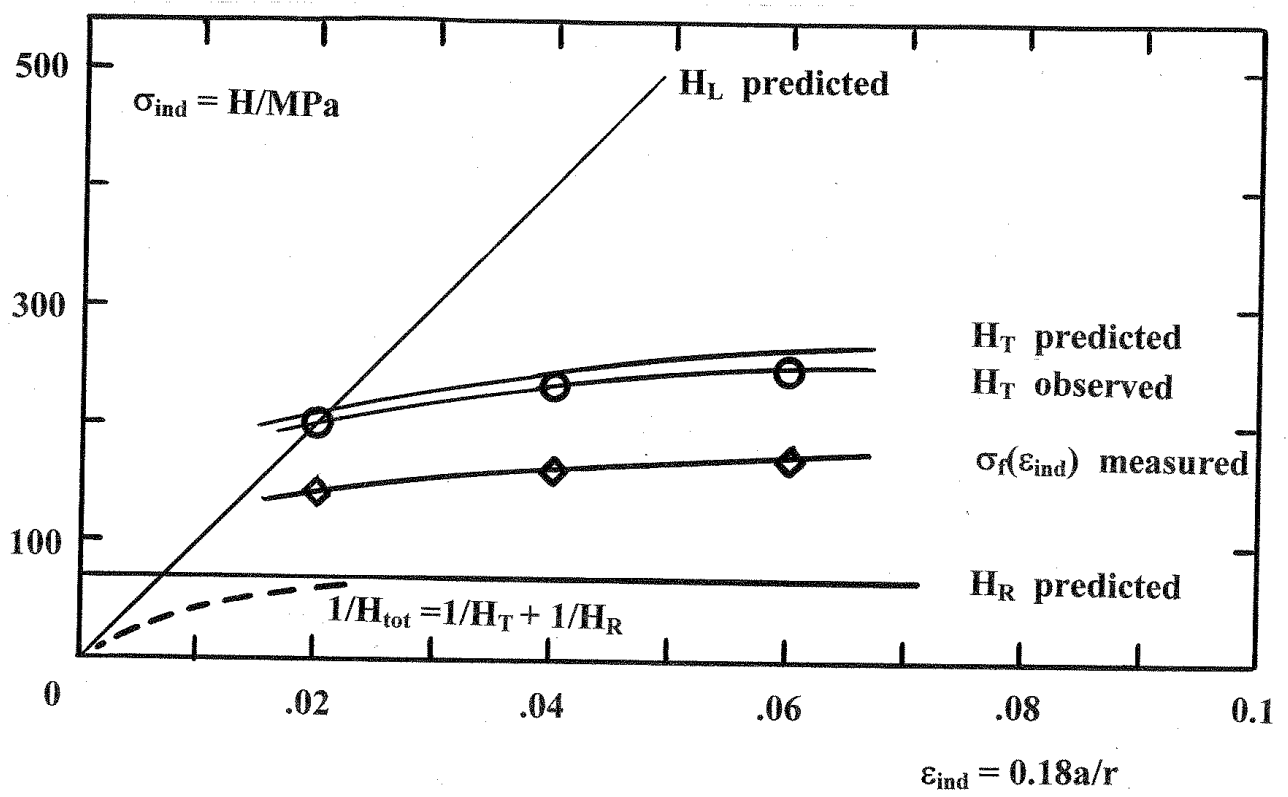

Fig. 6: Hardness as a function of representative strain for one indenter radius. Independent estimates of the various components produce a 'predicted' total hardness which can be compared with what is observed. 


$$
\frac{r}{b}>>\left(\frac{\mu}{\sigma(\varepsilon)}\right)^{2}
$$

The effects of the geometrically necessary dislocations are surprisingly large when one is working with contact sizes much less than the indenter diameter, that is, at very small representative strains. If the sample is a virgin pure ductile material, the tensile flow stress can be very small indeed, and an indenter of centimetre size may be needed. However, if a work-hardened sample has a flow stress nearer to $\mu / 1000$, an indenter of millimetre size should require negligible correction.

(2) The second example is taken from work using spherical indenters to characterise polycrystalline annealed electrodeposited copper $[13,14]$. To simplify the interpretation, the experiments were performed at constant representative strain, that is, at a constant value of $a / r=1 / 4$. A wide range of grain sizes overlapping with a wide range of indenter sizes gives a good linear relationship between the square of the hardness and an inverse effective length $l_{\text {eff }}$ found empirically by curve-fitting to be given by

$$
\frac{1}{l_{\text {eff }}}=\frac{1}{L}+\frac{8}{3 r}
$$

Fig. 7 shows the excellent comprehensive data. How well is this behaviour explained by the threedimensional versions of equations (4) and (5) given in Table 1? Firstly, it is clear that the geometrically necessary dislocation density due to the polycrystalline flow should be added to that due to rotational flow around the indenter. This produces a density

$$
\rho=\frac{3 A \varepsilon}{b L}+\frac{1}{b r}
$$

Quantitative agreement with experiment can be achieved by fitting an appropriate value of the strain $\varepsilon$ and the forest hardening constant $\alpha$. We find that agreement requires $\varepsilon \approx 0.24$ and $\alpha \approx 0.16$. The strain is approximately equal to five times the representative strain, a result consistent with the interpretation of Narutani and Takamura's results [11] noted earlier, and the value of $\alpha$, although somewhat low, is consistent with other values listed in Table 1.

(3) Observations of rotations using convergent beam electron diffraction in electron beam transmission through thin sections beneath a Berkovich indenter in copper [15] show a rotation field extending outwards from the edge of the indentation to a distance about equal to the indentation size itself, as expected. What is of great significance is that although the larger indent shows a maximum angle of rotation equal to the expected value within experimental error, the smaller indent shows a smaller angle, about two-thirds that value. This is very direct evidence, not dependent upon observations by etch pits, of the transition from rotational flow to laminar flow at smaller indents. Agreement between theory and experiment can be made semi-quantitative on the basis of a reanalysis of their data. This behaviour is not predicted by other theories. 


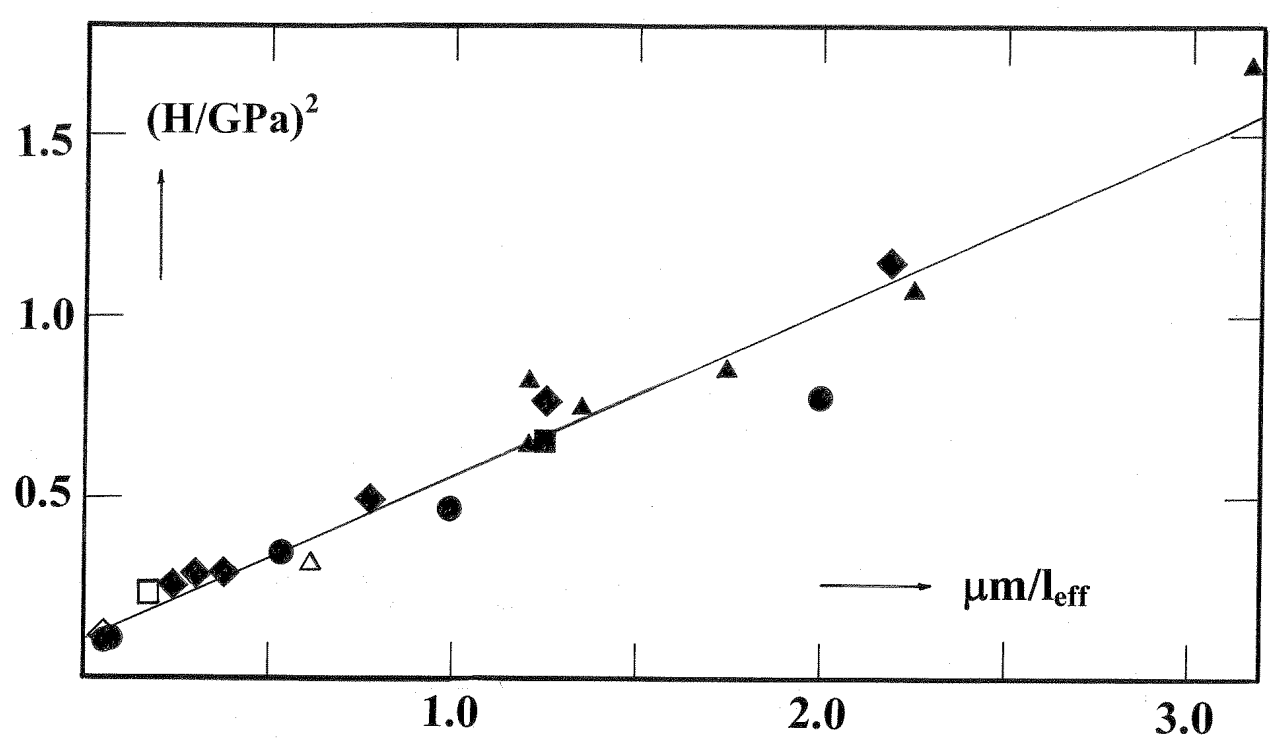

Fig. 7. Data replotted from refs. [13, 14] showing hardness of electrodeposited and annealed polycrystalline copper at constant representative strain, corresponding to $a / r=0.25$. The indenter radii are: filled triangles, $3 \mu \mathrm{m}$; filled squares, $5 \mu \mathrm{m}$; open triangles, $7 \mu \mathrm{m}$; filled diamonds, $21 \mu \mathrm{m}$; open squares, $30 \mu \mathrm{m}$; filled diamonds, $200 \mu \mathrm{m}$; open diamonds, $500 \mu \mathrm{m}$. The grain sizes vary from about one-third the indentation diameter to about twenty-five times the diameter. The best-fit straight line is given by $(H / G P a)^{2}=0.46\left(\mu m / l_{\text {eff }}\right)+.098$. The intercept corresponds to a tensile flow stress of about $100 \mathrm{MPa}$ at the representative strain of about $5 \%$, a not unreasonable value.

(4) A recent paper [20] demonstrates work-hardening caused by rolling nanocrystalline (NC) Ni, an observation of potential practical significance. Very strong linear hardening gives way to parabolic hardening at about 5\% rolling strain. This is readily interpretable as laminar flow producing internal stress followed by transgranular rotational flow. The 3-D version of Equation (5) can be tested at $10 \%$ strain and it predicts quite accurately the larger dislocation densities estimated by the authors using high resolution electron microscopy, but it overestimates considerably the average density and that obtained by X-ray diffraction. The density varies nearly linearly with strain, as expected. A value of $\alpha$ can be deduced; it is about 0.2 .

(5) There are now many papers presenting data on the indentation size effect. It seems appropriate to use the new data to determine the values of the forest-hardening parameter $\alpha$ relating to rotational flow and the Chaudhri constant $C$ relating to laminar flow. In the past, twodimensional formulae have been used to analyse the experimental results, and no systematic attempt has been made to determine these constants, except to note that they have values which seem reasonable. In the last column of Table 1 are shown the values of $\alpha$ and $C$ required to bring experiment and the 3-D theory into close agreement. Various uncertainties enter the determinations, not least the variability of surface orientation and the difficulty in some cases of assessing the weight of different contributions to the hardness. No attempt has been made to include results from materials which are not fully plastic and display cracking under indentation, such as $\mathrm{MgO}$. These 
materials generally show much more laminar flow than ductile metals, as one expects. They also show much larger apparent values of $\alpha$, for which there seems to be no simple explanation.

In summary: there are only two determinations of $C$, which are consistent with one another and suggest a value about 36. The 10 values of $\alpha$ cluster around 0.25 for a wide range of indenters and metals. The nominal value assumed for $\alpha$ in the past was $1 / 3$. The most authoritative study of the flow stress in shear as a function of the forest dislocation density is by Basinski and Basinski [21] who find a relationship complicated by the logarithmic dependence of the dislocation line tension upon the obstacle spacing. At the highest density encompassed by their data, one finds $\alpha \approx 0.6$, but at the very high dislocation densities relevant here one might anticipate $\alpha \approx 0.4$. A recent authoritative paper [22] analyses all data and computer models to find $\alpha \approx 0.2$ at high densities. It is interesting that the derived values in Table 1 seem to support this lower value.

\section{Residual elastic strain and the cohesive strength of polycrystals}

The Hall-Petch law is seen to be a close relative of the indentation size effect. The famous inverse square root relationship between grain size and flow stress will be apparent whenever the geometrically necessary dislocation density exceeds the background density, that is, at small grain sizes and moderate strains. In the neighbourhood of yield for virgin crystals, one expects plasticity to be controlled by other effects, such as the release of dislocations from grain boundaries or Cottrell atmospheres. Also, in highly work-hardened metals the geometrically necessary dislocations may be swamped. There is clear evidence for this [11]. At very small strains, one expects the plastic accommodation of grains in a polycrystalline array to occur by laminar flow with the build up of large internal stress, just as for indentation plasticity. Once again, there is much evidence which can be interpreted as a transition from laminar to rotational flow as strain increases, smaller grains requiring larger strains to make the transition.

It is surprisingly easy to estimate the residual stress in a polycrystal [10]. Regardless of whether the flow is laminar or rotational, the level of internal stress cannot exceed the local flow stress. In the strained state, the aggregate may have a tensile flow stress $\sigma$ but within the polycrystal there will be a random distribution of elastic stresses, averaging to zero in the unloaded state. There will be stress discontinuities across grain boundaries parallel to the tensile axis. At boundaries perpendicular to the tensile axis there will be residual tension or compression, continuous across them. If there is a random distribution of intergranular residual stress, ranging from $+\sigma$ to $-\sigma$, the energy density is given in terms of the Young modulus $E_{Y}$ by the mean square value $\left\langle\sigma^{2}\right\rangle / 2 E_{Y}=\sigma^{2} / 4 E_{Y}$. A crack running along the grain boundaries releases this stress, but only within a sandwich of material centred on the crack and of thickness approximately equal to the grain size. The elastic energy release rate is thus $\sigma^{2} / 4 L E_{Y}$. If this is greater than the work of adhesion at the boundaries, $W_{A D}$, cohesion of the aggregate is lost. On can thus estimate the maximum tensile stress supportable by the polycrystal without fracturing: it is

$$
\sigma_{\max } \approx \sqrt{\frac{4 E_{Y} W_{A D}}{L}} .
$$

The grain boundaries appear to act as Griffith cracks. Insertion of numerical values for grain boundaries in the absence of embrittling segregation shows that polycrystals are resistant to decohesion except at very large plastic strains, as one knows empirically. The model of an assembly of deformed grains as a collection of interpenetrating hardness indenters seems to provide a sound physical basis upon which to discuss the cohesion of polycrystalline arrays. 


\section{Relationship to earlier models}

Although in recent years the study of the indentation size effect has brought about a revolution in understanding of the hardness of metals, it is important to recollect that it is important only for relatively small indentations, less than a micron or so in size. The foundations laid by Tabor [23] and by engineering continuum mechanics as in Johnson's book [24] are remarkably sound. The principle that hardness is independent of indentation size is based mainly on the method of dimensions, in which the properties of the metal do not involve a length. However, the advent of dislocation theory with its introduction of the Burgers vector has changed that. The effects are larger than one might have thought, particularly for spherical indenters. It has been recognised for at least 50 years that smaller indentations show higher hardness [25] but uncertainties dogged the discussions, particularly concerning surface preparation and the reliability of measurement when the indent is itself smaller than the wavelength of light.

The advent of reliable instrumented indentation techniques with sub-Angstrom resolution [26] and of microscopes capable of atomic resolution, STM and AFM, has changed the situation. Perhaps the first modern paper showing the indentation size effect using instrumented indentation under a Berkovich indenter in Al is that by G. N. Kalei [27]. However, this early paper seems to have been ignored in the west. It is also the case that instrumented indentation performed without microscopical observation is subject to error which may come from unknown roundedness on a nanometre scale at the nominally sharp tip of the indenter [28]. However, with continuing reduction in the size of mechanical components, the indentation size effect is of potential engineering importance, as well as bringing about a profound change in the theories of plasticity.

The notion that there is a transition from laminar to rotational flow under an indenter is certainly not new: a summary of related work from the Moldovian Academy of Sciences [29] indicates that the rotation mechanism has for some years been viewed as a disclination process, operating at the mesolevel, in contrast to the dislocation (laminar) process at the microlevel. Also the view of Chaudhri [4] that flow occurs first into the material and then upwards towards the surface is well established, particularly for ceramics like $\mathrm{MgO}$. Authors who have observed the striking rosette structures in a wide variety of crystalline materials including metals tend to be reluctant to accept the picture of plastic flow suggested by plasticity theory and often ignore it. The present model can be viewed as an attempt to synthesize these various points of view.

\section{Acknowledgements}

I am indebted to Dr. Munawar Chaudhri for an invitation to present this paper at a one-day conference organised to mark his retirement; to Prof. Feodor Borodich for showing me the earlier Russian work; also, as usual, to the Cavendish Laboratory and to Robinson College for support. 


\section{References}

[1] M.F. Ashby: Phil. Mag. Vol. 21 (1970) p. 399.

[2] N.A. Fleck and J.W.Hutchinson: J. Mech. Phys. Sol. Vol. 41 (1994) p. 1825; also, Adv. Appl. Mech., Vol. 33 (1997) p. 295.

[3] L.M. Brown: Slip circle constructions for inhomogeneous rotational flow, Materials Science Forum Vol. 550 (2007) pp $105-107$.

[4] M.M. Chaudhri: Dislocations and Indentation, Chapter 12 in Dislocations in Solids, F.R.N. Nabarro and J.P. Hirth, Eds., Elsevier B.V., (1994).

[5] H.Czerski and L.M. Brown: J. Phys. D.: Applied Physics Vol 41 (2008) pp 1 - 5.

[6] D. Tabor, The Hardness of Metals (1951), Clarendon Press, Oxford; paperback edition (2000).

[7] N.A. Stelmashenko, M.G. Walls, L.M. Brown, and Yu.V. Milman, Acta metal. mater., Vol 41 (1993) pp. $2855-2865$.

[8] J. G. Swadener, E. P. George. And G. M. Pharr: J. Mech. Phys. Sol. Vol 50 (2002) p 681.

[9] Yong Lee Lim and M. Munawar Chaudhri: Phil. Mag. Vol A 79 (1999) p. 2979.

[10] L.M. Brown, in 'Plasticity, Failure and Fatigue in Structural Materials - from Macro to Nano', Proceedings of the Hael Mughrabi Honorary Symposium, (2008) Eds. E. Jimmy Hsia, Mathias Göken, Tresa Pollock, Pedro Dolabella and Neville R. Moody, published by TMS, Warrendale Pa, pp $105-117$. The values of the averaged Schmidt factors given here have been corrected from those in the paper cited.

[11] T. Narutani and J. Takamura: Acta metall. mater. Vol 39 (1991) pp 2037 - 2049.

[12] Fuquian Yang, Lingling Peng, and Kenji Okazaki; Materials Characterization Vol 57 (2006) pp $321-325$

[13] X. Hou, A.J. Bushby, and N.M. Jennet; J. Phys. D: Applied Physics Vol 41 (2008) 074006 $(7 \mathrm{pp})$

[14] X. Hou; Geometrical Size Effects in the Plasticity of Metals by Micromechanical Testing: $\mathrm{Ph}$.D. thesis for Queen Mary College, University of London, 2009.

[15] K. K. McLaughlin and W. J. Clegg; J. Phys. D: Applied Physics Vol 41 (2008) 074007 (5pp)

[16] N. Stelmashenko; Microstructual Studies of Plastic Indentations at Low Loads: Ph.D. thesis for the University of Cambridge, 1995.

[17] N. Gane and J. M. Cox; Phil. Mag. Vol 22 (1970) p 881

[18] Pethica, J. B., Hutchings, R. and Oliver, W. C.; Phil. Mag. Vol A48 (1983) p 593.

[19] Y. H. Lee, J. H. Hahn, S. H. Nahm, J. I. Jang, and D. Kwon; J. Phys. D: Applied Physics Vol $41(2008) 074027$ (5pp). 
[20] X. L. Wu, Y. T. Zhu, Y. G. Wei, and Q. Wei; Phys. Rev. Lett. Vol 103 (2009) 205504 (4pp)

[21] Z. S. Basinski and S. J. Basinski; Dislocations in Solids Vol. 4 (1979), F. R. N. Nabarro, Ed., (North Holland, Amsterdam) p 26.

[22] R. Madec, B. Devincre, and L. P. Kubin, Phys. Rev. Lett. Vol. 89 (2002) 255508 (4pp).

[23] D. Tabor, The Hardness of Metals, Oxford University Press, New York and Oxford, 1951, reissued as a paperback in the Oxford Classics Series, 2000.

[24] K. L. Johnson; Contact Mechanics, Cambridge University Press, Cambridge U.K., New York, Melbourne, 1985.

[25] B. W. Mott, Microindentation Hardness Testing, Butterworth, London, 1956.

[26] Oliver, W. C. and Pharr, G. M.; Journal of Materials Research Vol 7, (1992), p1564; also Vol 19 (2004) p 3.

[27] Kalei, G. N.; Mashinovedenie Vol 4, (1968) p 105 (in Russian)

[28] Feodor M. Borodich and Leon M.Keer; International Journal of Solids and Structures, Vol 41 (2004) p 2479. This article reviews work on tip-shape functions.

[29] D. Grabco, O. Shikimaka, and E. Harea; J. Phys. D. Appl. Phys. Vol 41 (2008) 074016 (9pp) 